\title{
Análisis factorial confirmatorio del Cuestionario SA-45 en una muestra mexicana
}

\author{
Blanca G. Alvarado ${ }^{1}$, Bonifacio Sandín²*, José L. Valdez-Medina ${ }^{1}$, Norma González-Arratia ${ }^{1}$, Sofía Rivera ${ }^{3}$
}

${ }^{1}$ Universidad Autónoma del Estado de México (México)

${ }^{2}$ Universidad Nacional de Educación a Distancia, Madrid (España)

${ }^{3}$ Universidad Nacional Autónoma de México (México)

\begin{abstract}
Resumen: El objetivo de la presente investigación consistió en examinar la estructura factorial, la fiabilidad y los datos normativos del Cuestionario SA-45 (Symptom Assessment-45) en una muestra de estudiantes universitarios mexicanos. Como se predijo, los análisis factoriales confirmatorios indicaron que el modelo de nueve factores proporcionaba el mejor ajuste del SA-45. Así mismo, los datos demostraron buenos niveles de fiabilidad (consistencia interna) del cuestionario. Los datos normativos mostraron que las mujeres puntuaban más alto que los varones en todas las escalas excepto en psicoticismo. En general, el estudio proporciona apoyo transcultural consistente sobre la fiabilidad y validez del cuestionario.

Palabras clave: SA-45; SCL-90; síntomas; psicopatología; evaluación; autoinforme.
\end{abstract}

\section{Introducción}

El uso de cuestionarios de autoinforme constituye un recurso fundamental en la cuantificación de variables psicopatológicas, ya que son imprescindibles para evaluar la introspección que subyace a cualquier sintomatología y es consustancial a esta misma. Aunque la mayor parte de los instrumentos clínicos de autoinforme se han diseñado para la evaluación de constructos psicopatológicos más o menos específicos (e.g., Belloch, Reina, García-Soriano y Clark, 2009; Pilatti, Godoy y Brussino, 2010; Sandín, Chorot, Santed y Valiente, 2002; Sandín, Chorot, Valiente y Lostao, 2009), también se han utilizado para determinar la sintomatología de aspectos más globales del individuo (e.g., Derogatis, 1990; Fonseca, Paino, Lemos y Muñiz, 2011; Sandín, Chorot y Valiente, 2009). El Symptom Checklist 90 (SCL-90; Derogatis, 1990, 1994; Derogatis, Lipman y Covi, 1973), en sus diversas revisiones, es uno de los instrumentos de autoinforme de evaluación global de psicopatología más utilizado a nivel internacional, habiendo sido empleado con éxito en la investigación y en la práctica clínica para diferenciar entre diversas manifestaciones o trastornos psicopatológicos, para evaluar los cambios terapéuticos, en estudios de cribado, en investigaciones sobre calidad de vida, y para obtener indicadores globales de gravedad psicopatológica (Derogatis, 1994; Gempp y Avendaño, 2008; Hard, Gerbershagen y Franke, 2000; Jin et al., 2010; López, Fernández y Becoña, 2009; Strauman y Wetzler, 1992; Sandín, Chorot, Valiente, Lostao y Santed, 2002; Schmitz et al., 2000; Strauman y Wetzler, 1992). También ha sido estudiado extensamente en atención primaria con diferentes finalidades clínicas y de investigación, incluidas las relacionadas con el impacto de la morbili-

* Dirección para correspondencia [Correspondence address]: Bonifacio Sandín, Universidad Nacional de Educación a Distancia, Facultad de Psicología, Juan del Rosal 10, 28040 Madrid (España). E-mail: bsandin@psi.uned.es
Title: Confirmatory factor analysis of the SA-45 Questionnaire in a Mexican sample.

Abstract: The aim of this work was to examine the factor structure, reliability and normative data of the Symptom Assessment-45 Questionnaire (SA-45) in a Mexican sample of undergraduates. As predicted, confirmatory factor analyses indicated that a multidimensional 9-factor model provided a good fit for the SA-45. Data on reliability (internal consistency) show the SA-45 to have sound psychometric properties. Normative data suggest that women scored higher than men on all scales except psychoticism. Overall, the study provides consistent cross-cultural support for reliability and validity of the questionnaire.

Key words: SA-45; SCL-90; symptoms; psychopathology; assessment; self-report.

dad psicológica en los pacientes con enfermedades médicas, la duración de la hospitalización, el nivel de perturbación psicológica, y la comorbilidad en pacientes psiquiátricos (Derogatis y Savitz, 2000).

El SCL (hasta su forma actual de 90 items) se elaboró a partir del Cornell Medical Index (Wider, 1948), así como mediante criterios empíricos (experiencia clínica) y psicométricos, es decir, empleando tanto criterios clínicos como estadísticos. La forma más común del cuestionario es la constituida por 90 items (Derogatis, 1994), la cual incluye los 9 factores (escalas) siguientes de sintomatología psicopatológica: depresión, hostilidad, sensibilidad interpersonal, somatización, ansiedad, psicoticismo, obsesión-compulsión, ansiedad fóbica (agorafobia), e ideación paranoide. Tanto el SCL90 como su forma revisada presentan ciertos problemas para su aplicación rutinaria que han sido señalados en la literatura, entre los que se encuentran los siguientes: elevado solapamiento entre items, baja validez discriminante, desequilibrio de las escalas en el número de items (las escalas varían entre 6 y 13 items), elevada correlación entre las escalas, y falta de validez factorial (los diversos estudios sobre la estructura del cuestionario han obtenido resultados que sugieren estructuras dispares, variando entre 1 factor y 10 factores primarios) (Bados, Balaguer y Coronas, 2005; Davison et al., 1997; Vassend y Skrondal, 1999; Zack, Toneatto y Streiner, 1998).

Aparte de estos problemas, el SCL-90 tiene el inconveniente de consistir en una prueba excesivamente larga, lo cual dificulta su utilización en aplicaciones rutinarias, en situaciones que exigen bajo consumo de tiempo, y en contextos clínicos o de investigación que requieren la evaluación de múltiples constructos psicológicos. Los instrumentos breves de evaluación, que permitan evaluar síntomas psicopatológicos y puedan ser empleados como herramientas de cribado y para evaluar los resultados terapéuticos, tienen actualmente una gran demanda y son de gran interés en psicopatología y psicología clínica (Sandín, Chorot, Valiente y Chorpita, 2010). La utilidad del SCL-90 podría incrementarse mediante 
la disponibilidad de una forma mejorada y más breve. En línea con esta idea, Davison et al. (1997) desarrollaron el Symptom Assessment-45 Questionnaire (SA-45), el cual constituye una forma abreviada del SCL-90. El SA-45, manteniendo las mismas dimensiones (escalas) y virtudes del SCL-90, incorpora las siguientes mejoras: (a) implica una reducción significativa del número de items del cuestionario, (b) iguala las escalas a un mismo número de items (5 items) y maximiza la posibilidad de que posean similar fiabilidad, (c) reduce el solapamiento entre las escalas (al haberse reducido los items redundantes), y (c) favorece la utilidad y uso del cuestionario. Se han proporcionado datos psicométricos sobre el SA-45, los cuales tienden a apoyar la estructura de 9 dimensiones, así como niveles apropiados de fiabilidad y validez convergente y discriminante (véase, Davison et al., 1997; Maruish, Bershadsky y Goldstein, 1998). Aunque aún se ha aplicado mucho menos que su antecesor, el SA-45 ha demostrado su validez en diferentes contextos y aplicaciones, entre los que se incluyen la clasificación y predicción de los pacientes psiquiátricos, la evaluación del cambio psicopatológico en pacientes con problemas de salud, los estudios sobre cribado psicopatológico (e.g., en estudios epidemiológicos), y la capacidad para reflejar los efectos del tratamiento psicológico en pacientes psiquiátricos (Maruis, 2000).

Recientemente Sandín, Valiente, Chorot y Santed (2008) llevaron a cabo una validación de la versión española del SA45, empleando una muestra amplia de estudiantes universitarios. Para probar la estructura del cuestionario, los autores llevaron a cabo análisis factoriales exploratorios, aplicando tanto análisis de componentes principales como análisis de ejes principales. Para la extracción del número de factores, Sandín et al. (2008) tuvieron en cuenta los siguientes criterios teóricos y/o empíricos: (1) Modelo teórico sobre la estructura de 9 dimensiones básicas, (2) evidencia de otras posibles estructuras encontradas a partir del SCL-90 (entre $1 \mathrm{y}$ 10 factores), (3) criterio de Kaiser, (4) test de scree (Cattell, 1966), y (5) interpretabilidad de las diferentes estructuras factoriales resultantes (Gorsuch, 1983), que implica examinar soluciones con diferentes criterios de extracción para determinar el punto en el que aparecen los factores triviales o redundantes. Los autores, aparte de emplear el método de componentes principales para la extracción de los factores, necesario para poder contrastar los estudios factoriales efectuados con el SCL-90 y SA-45, aplicaron también el método de ejes principales. En ambos casos, Sandín et al. (2008) obtuvieron una estructura de 9 factores correlacionados que correspondían a las nueve subescalas propuestas en la versión original del cuestionario (Davison et al., 1997). Los autores presentaron los resultados de la rotación oblicua (promax), ya que asumían que los factores deberían correlacionar entre sí. Como habían hipotetizado, los autores obtuvieron correlaciones moderadas entre los factores.

La estructura factorial fue replicada por los autores mediante análisis factoriales confirmatorios (Sandín, et al., 2008), sugiriendo que la estructura que mejor se ajustaba a los datos era la de nueve factores correlacionados, al con- trastarla con una estructura de un solo factor (factor general de psicopatología) y con un modelo jerárquico de nueve factores primarios y dos factores de orden superior (neurosis y psicosis). Concluyeron que el SA-45, aparte de su valor como medida general de psicopatología, posee escalas separadas válidas y fiables que pueden ser de utilidad para diferenciar entre distintos tipos de trastornos y/o facetas diferentes de la psicopatología. El SA-45 presenta sobre el SCL-90-R las ventajas de ser más breve, y posiblemente más discriminativo, puesto que se ha mejorado la validez y consistencia de las dimensiones (Sandín et al., 2008).

Los autores proporcionaron evidencia a favor de la fiabilidad (consistencia interna) del cuestionario, con un coeficiente alfa de Cronbach para el cuestionario total de 0,95, y con coeficientes alfa de las subescalas que, con la excepción de la escala de psicoticismo, todos superaban el valor de 0.70 (variaron entre 0.71 y 0.85 ). Las correlaciones entre las escalas variaron entre 0.73 y 0.39 , inferiores a las que se han venido señalando para las escalas del SCL-90 (Strauman y Wetzler, 1992). Sandín et al. (2008) también aportaron datos sobre la validez convergente y discriminante del SA-45, a través de las correlaciones obtenidas entre las escalas de este cuestionario y otros constructos cercanos o divergentes con las dimensiones del SA-45.

La versión española del SA-45 se ha empleado recientemente en estudios en el ámbito clínico. Así por ejemplo, Merino (2010) constató que el instrumento resultaba útil para detectar problemas psicopatológicos en individuos con elevados niveles de estrés laboral y/o síndrome de burnout. En una línea similar, Delclaux (2011) aplicó este instrumento en pacientes con diagnóstico de cáncer sometidos a quimioterapia, encontrando alteraciones significativas en algunas de las subescalas (especialmente en las escalas de depresión, somatización, ansiedad y obsesión-compulsión). También se está aplicando a nivel internacional en investigación psicopatológica, tal y como se evidencia, por ejemplo, a través del estudio llevado a cabo por Grill, Posada y Castañeiras (2009) sobre los efectos psicopatológicos de las situaciones traumáticas.

Dado el interés que suscita el SA-45 por sus posibles ventajas sobre otras formas derivadas del SCL-90, sería importante llevar acabo estudios basados en poblaciones de otros países con objeto de poder contrastar transculturalmente sus características psicométricas. Mediante el presente estudio examinamos las propiedades psicométricas de la versión española del SA-45 en una población de estudiantes mexicanos. El primer objetivo se centra en examinar mediante análisis factorial confirmatorio la estructura del cuestionario previamente sugerida por Sandín et al. (2008). En relación con este objetivo hipotetizamos que debería confirmarse la estructura de nueve factores correlacionados referidos a las nueve dimensiones del cuestionario, debiendo ser esta estructura más consistente que las estructuras alternativas de un factor general o de dos factores de orden superior (neurosis y psicosis) y nueve factores primarios (estructura jerárquica). El segundo objetivo fue estudiar la fiabilidad 
(consistencia interna) del cuestionario, hipotetizando que tanto los coeficientes alfa como las correlaciones ítem-escala deberían indicar valores aceptables o buenos. De acuerdo con Nunnally (1978), suelen considerarse aceptables los valores a partir de 0.70 , y buenos los valores a partir de 0.80 . En lo que concierne a la correlación ítem-escala, el menor nivel aceptable suele ser de 0.20 (Nunnally, 1978). Finalmente, intentamos obtener información preliminar sobre datos normativos del cuestionario para la población mexicana.

\section{Método}

\section{Participantes}

Utilizamos una muestra de 418 estudiantes universitarios de la Universidad Autónoma del Estado de México. Doscientos dos (48.3\%) eran mujeres y 216 hombres (51.7\%). Las edades de los participantes oscilaron entre 18 y 55 años con una media de 20.9 años $(D T=2.3)$ (grupo de mujeres) y $21.3(D T=2.9)$ (grupo de hombres). La mayor parte de los participantes eran de nivel económico medio (89\%).

\section{Instrumentos de evaluación}

SA-45 (Symptom Assessment-45 Questionnarie) (Davison et al., 1997). Empleamos la versión española validada por Sandín et al. (2008). El cuestionario evalúa las mismas dimensiones que el SCL-90-R: hostilidad, somatización, depresión, obsesión-compulsión, ansiedad, sensibilidad interpersonal, ansiedad fóbica, ideación paraniode y psicoticismo. Los participantes deben contestar cada ítem señalando la frecuencia con la que haya experimentado cada uno de los 45 síntomas durante la última semana, entre 0 ("nada en absoluto") y 4 ("mucho o extremadamente"). Se ha referido evidencia en apoyo de su fiabilidad y validez, tanto para la versión inglesa (Davison et al., 1997) como para la versión española (Sandín et al., 2008).

\section{Procedimiento}

A los participantes se les informó previamente sobre los objetivos del estudio y las recomendaciones sobre la forma de responder al cuestionario. Contestaron en forma colectiva (aproximadamente en grupos de 10 a 25 personas) en los centros educativos. La participación fue voluntaria y anónima con consentimiento informado; se garantizó la confidencialidad de la información. El protocolo de investigación fue aprobado por el comité de ética de la Universidad Autónoma del Estado de México.

\section{Análisis estadístico}

En el presente estudio hemos aplicado análisis factoriales confirmatorios (AFC), y análisis de varianza multivariado y univariados. También calculamos los coeficientes de fiabilidad (alfa de Cronbach y correlación ítem-escala corregida) para los diferentes items y/o escalas del SA-45. Finalmente se calcularon los datos normativos (medias, DTs y percentiles). Determinamos los percentiles (puntos de corte) para las nueve escalas del SA-45 y para la puntuación total en el cuestionario, separando los valores correspondientes a los grupos de varones y mujeres.

Mediante la aplicación de análisis factoriales confirmatorios (AFC) se pretendió probar la adecuación de la estructura de nueve factores obtenida en otras investigaciones (Sandín et al., 2008), así como otras posibles estructuras sugeridas previamente para el SCL-90. En concreto, se probaron los tres modelos competitivos siguientes: (1) modelo de nueve factores correlacionados; (2) modelo de un solo factor; y (3) modelo de nueve factores primarios y dos factores de orden superior, denominados éstos "distress" (incluye las dimensiones: depresión, somatización, ansiedad, obsesióncompulsión, y ansiedad fóbica) y "psicosis" (incluye las dimensiones: hostilidad, psicoticismo e ideación paraniode). La dimensión de sensibilidad interpersonal es una dimensión más inespecífica y no se ha asimilado separadamente a ninguno de estos dos factores de orden superior, por lo cual tampoco fue incluida en nuestro análisis jerárquico.

La inclusión del modelo basado en un factor único obedece a la evidencia referida por algunos estudios con el SCL90 que sugiere la existencia de un factor general de psicopatología (Carlozzi y Long 2008), más que varios factores específicos. Asimismo, la inclusión del tercer modelo se basa en algunos datos sobre la estructura del SCL-90 que sugieren dos grandes factores de orden superior, uno relacionado con la ansiedad y la depresión ("distress") y otro con el pensamiento paranoide ("psicosis") (Arrindell, Barelds, Janssen, Buwalda, y van der Ende, 2006; Bados et al., 2005).

Los análisis confirmatorios se realizaron con la muestra total y se empleó el programa de ecuaciones estructurales EQS (Bentler, 2005). Se contrastaron los tres modelos indicados. Para la identificación del modelo de nueve factores (indicadores de las variables latentes) nos basamos en los resultados del análisis factorial exploratorio de Sandín et al. (2008), según el cual cada variable latente (factor) estaba definida por 5 variables de medida específicas (indicadores). La identificación del modelo de un factor se realizó con los 45 ítems del cuestionario, los cuales deberían saturar en un único factor. Finalmente, el modelo tercero incluía nueve factores primarios identificados igual que en el modelo 1, y dos factores de segundo orden identificados a partir de las cinco variables latentes de ansiedad, depresión, obsesióncompulsión, ansiedad fóbica y somatización (factor de "distress") y las tres variables latentes de hostilidad, psicoticismo e ideación paranoide (factor de "psicosis"). Las varianzas de los términos de error fueron especificadas como parámetros libres. No se permitió la covariación entre los residuos. Puesto que los datos basados en cuestionarios tipo Likert pueden presentar problemas de distribución normal por tratarse de variables no continuas, se utilizó el método de estimación ML-robusto, el cual proporciona estadísticos robustos, incluida la prueba escalada de $\chi^{2}$ de Santorra-Bentler, co- 
rrigiendo los errores debidos a una posible violación del principio de normalidad.

De acuerdo con Brown (2006), para la determinación del ajuste del modelo nos guiamos por un conjunto de índices de bondad de ajuste (incluidos los tipos descriptivo, absoluto, de parsimonia y comparativo), haciendo una valoración global del conjunto de índices, más que centrarnos en el punto de corte de un determinado índice. Se usaron los siguiente indicadores de bondad de ajuste: (1) $\chi^{2}$ dividido por los grados de libertad (cocientes $\leq 2.0$ indican excelente ajuste; a menor índice menor ajuste; Bollen, 1989); (2) CFI (comparative fit index); (3) SRMR (standardized root meansquare residual); (4) RMSEA (root mean square error of approximation); y (5) AIC (Akaike's information criterion). Se considera un ajuste aceptable si los valores de CFI se acercan a 0.90 (Bentler, 1992), SRMR $\leq 0.08$ (Hu y Bentler, 1999), y RMSEA $\leq 0.06$ (Hu y Bentler, 1999). El AIC es un indicador relativo; en ausencia de otros criterios definitorios, el mejor modelo es el que posea un AIC más bajo (Bentler, 2005).
Para examinar las posibles diferencias en las puntuaciones a las escalas en función del sexo, se aplicó inicialmente un análisis de varianza multivariado de un factor (sexo) y nueve variables dependientes (las nueve escalas del SA-45). Posteriormente se aplicaron análisis de varianza univariados para calcular los efectos separados de la variable sexo sobre cada una de las nueve variables dependientes.

\section{Resultados}

\section{Análisis factorial confirmatorio del SA-45}

Los datos correspondientes a los análisis factoriales confirmatorios aparecen indicados en la Tabla 1. A partir de los datos que incluimos en esta tabla puede observarse que el modelo que mejor se ajusta a los datos es el modelo 1 (nueve factores correlacionados). Este modelo es superior a los dos restantes en todos los indicadores de ajuste. Asimismo, el valor de AIC es más bajo que para los restantes modelos.

Tabla 1. Índices de bondad de ajuste para los diferentes modelos sobre la estructura del SA-45, basados en los análisis factoriales confirmatorios EQS ( $N=418$ ).

\begin{tabular}{|c|c|c|c|c|c|}
\hline Modelo del SA-45 & $\chi^{2 / g l}$ & CFI & SRMR & RMSEA & AIC \\
\hline Modelo 1: Nueve factores correlacionados & 1.7 & 0.85 & 0.06 & 0.04 & -278.4 \\
\hline Modelo 2: Un factor & 2.4 & 0.66 & 0.07 & 0.06 & 388.6 \\
\hline Modelo 3: Modelo jerárquico & 4.9 & 0.77 & 0.21 & 0.05 & -4.9 \\
\hline
\end{tabular}

Nota: $\mathrm{CFI}=$ comparative fit index; $\mathrm{SRMR}=$ standardized root mean square residual; RMSEA= root mean error of approximation; $\mathrm{AIC}=$ Akaike's information criterion

Tanto el modelo de un factor como el modelo jerárquico se ajustan a los datos de forma mucho más pobre, sobre todo por los bajos índices del CFI, así como también por el elevado valor de la SRMR en el modelo 3. En relación con los índices de ajuste del modelo 1 cabe decir que, si bien el valor del CFI no es bueno (está próximo al valor aceptable), los restantes índices son excelentes. En la Tabla 2 indicamos los valores de las estimaciones paramétricas de las ecuaciones de medida (coeficientes completamente estandarizados) para cada uno de los items del SA-45, constatándose valores elevados para la mayoría de los items.

\section{Fiabilidad del SA-45}

En la Tabla 2 se indican los coeficientes alfa de Cronbach para cada una de las escalas del cuestionario, siendo la mayoría cercana o superior a 0.80 (i.e., niveles buenos de consistencia interna). Sólo una escala (Psicoticismo) evidenció un valor inferior a 0.70 , denotando un nivel bajo de fiabilidad (consistencia interna). El coeficiente alfa para el SA-45 total fue de 0.96. Los coeficientes son similares a los referidos por Davison et al. (1997) para la versión inglesa del cuestionario y por Sandín, et al. (2008) para la versión española. Al igual que obtuvieron estos autores, el coeficiente más bajo corresponde a la escala de psicoticismo. Cabe resaltar que, salvo esta circunstancia, los coeficientes alfa para las diferentes escalas fueron en general buenos, a pesar del limitado número de items de cada escala (5 items).
Las correlaciones corregidas ítem-escala están indicadas en la Tabla 2. Salvo para los items 1 ("La idea de que otra persona puede controlar sus pensamiento") y 4 ("Oír voces que otras personas no oyen") del factor psicoticismo, las correlaciones tienden a ser entre moderadas y altas, lo que sugiere que los elementos representan apropiadamente a las escalas correspondientes (todas las correlaciones fueron superiores a 0.20 ). Las correlaciones más altas corresponden a las escalas más consistentes factorialmente (depresión, hostilidad, etc.).

Las correlaciones entre las escalas variaron entre 0.76 y 0.34 , lo que indica que, aun siendo entre moderadas y altas, son similares a las obtenidas por Sandín et al. (2008) e inferiores a las que se han venido refiriendo para las escalas del SCL90-R (véase por ejemplo, Barker-Collo, 2003). Las correlaciones más elevadas se obtienen para la escala de Sensibilidad interpersonal (con Depresión $=0.76$; con Obsesión compulsión $=0.73$ y con Ideación paraniode $=0.72)$ y para la escala de Depresión (con Ansiedad $=0.74$ y con Obsesión-compulsión $=0.71)$. Las correlaciones más bajas se obtienen para la escala Ansiedad fóbica (con Hostilidad = 0.34; con Somatización $=0.38$ y con Psicoticismo $=0.36$ ). Estos resultados son consistentes con los obtenidos por Davison et al. (1997) y Sandín et al. (2008) sobre las elevadas correlaciones de la sensibilidad interpersonal con otras variables del cuestionario, debido tal vez a un problema de validez de contenido de la primera. 
Tabla 2. Factores del SA-45: Estimaciones paramétricas y correlaciones corregidas ítem-escala $(N=418)$.

$\begin{array}{ll}\text { Ítem del SA-45 (abreviado) } & \text { Estimación paramétrica }\end{array}$

Factor 1: Depresión ( $a=0.85)$

9. Sentirse $\operatorname{solo}(\mathrm{a})$

10. Sentirse triste

11. No sentir interés por las cosas

0.72

0.76

27.Sentirse desesperanzado con respecto al futuro

0.70

0.68

42. La sensación de ser un/a inútil

Factor 2: Hostilidad ( $a=0.85$ )

7. Arrebatos de cólera o fúria

34. Sentir impulsos agresivos

35. Tener ganas de romper algo

39. Discusiones frecuentes

43. Gritar o tirar cosas

Factor 3: Sensibilidad Interpersonal ( $a=0.85$

14. Sentirse incomprendido/a

15. Impresión de rechazo de otras personas

17. Sentirse inferior

32. Sentirse incómodo/a con la gente

Factor 4: somatización $(a=0.79)$

18. Dolores musculares

23. Calor o frio repentinos

26. entumecimiento o hormigueo

29. Debilidad corporal

31. Sensación de pesadez

Factor 5: Ansiedad ( $a=0.82$ )

6. Miedo repentino

12. Sentirse nervioso/a

30. Preocupación o tensión

38. Ataque de temor o pánico

41. Sentirse inquieto/a

Factor 6: Psicoticismo ( $a=0.57$ )

1. Creer que le controlan el pensamiento

4. oír voces que no oyen otros

13. Creer que otros conocen sus pensamientos

33. Pensamientos ajenos

45. Pensamientos de autocastigo

Factor 7: Obsesión-Compulsión $(\alpha=0.81)$

16. Tener que hacer las cosas muy despacio

20. Comprobación

21. Dificultad para tomar decisiones

28. Dificultad para concentrarse

3. Miedo a espacios abiertos

22. Miedo a viajar en medios de trasporte colectivo

Factor 9: Ideación Paranoide ( $a=0.74$ )

2. Sus problemas son culpa de otros 


\section{Datos normativos}

Las medias y desviaciones típicas se indican de forma separada para los grupos de hombres y mujeres (Tabla 3). Las puntuaciones en cada escala se obtuvieron sumando las puntuaciones en cada uno de los ítems que componen la misma (rango: 0-20). Las puntuaciones más elevadas corresponden a las escalas de Obsesión-Compulsión, Depresión y Ansiedad, y las más bajas a Psicoticismo y Ansiedad Fóbica, lo que concuerda con los resultados obtenidos en investigaciones anteriores con el SA-45 (Davison et al., 1997; Sandín et al., 2008).

Tras la aplicación del análisis de varianza multivariado obtuvimos efectos significativos globales [Hotelling $F$ $\left.(9.408)=7.23, p<.001, \eta_{p}{ }_{p}=.14\right]$, sugierendo un efecto significativo de la variable sexo sobre el conjunto de variables del SA-45.
Tabla 3. Medias y desviaciones típicas (DT) para escalas y total del SA-45 en función de los grupos de varones $(N=216)$ y mujeres $(N=202)$.

\begin{tabular}{|c|c|c|c|c|c|}
\hline \multirow[b]{2}{*}{$\begin{array}{l}\text { SA-45 (escalas: } \\
\text { rango } 0-20)\end{array}$} & Total & Varones & \multicolumn{3}{|l|}{ Mujeres } \\
\hline & $\begin{array}{l}\text { Media } \\
(D T)\end{array}$ & $\begin{array}{l}\text { Media } \\
(D T)\end{array}$ & $\begin{array}{l}\text { Media } \\
(D T)\end{array}$ & $F(1,418)$ & $\eta_{p}^{2}$ \\
\hline Depresión & $3.40(4.0)$ & $2.22(2.7)$ & $4.34(4.5)$ & $29.9 * * *$ & 0.07 \\
\hline $\begin{array}{l}\text { Hostilidad } \\
\text { Sensibilidad }\end{array}$ & $2.19(3.4)$ & $1.77(3.1)$ & $2.52(3.7)$ & $4.79 *$ & 0.02 \\
\hline Interpersonal & $2.84(3.6)$ & $1.98(2.7)$ & $3.52(4.0)$ & $19.49 * * *$ & 0.05 \\
\hline Somatización & $2.89(3.3)$ & $1.95(2.4)$ & $3.64(3.7)$ & $28.25^{* * *}$ & 0.06 \\
\hline Ansiedad & $3.35(3.7)$ & $2.09(2.7)$ & $4.36(4.1)$ & $41.68^{* * *}$ & 0.09 \\
\hline Psicoticismo & $1.28(1.9)$ & $1.15(1.7)$ & $1.39(2.1)$ & 1.58 & 0.00 \\
\hline Obse & & & & & \\
\hline Com 1 & $4.03(3.9)$ & $2.97(3.1)$ & $4.87(4.2)$ & $25.53^{* * *}$ & 0.06 \\
\hline Ansiedad Fóbica & $1.51(2.8)$ & $0.85(1.8)$ & $2.03(3.2)$ & $19.08 * * *$ & 0.05 \\
\hline Idea & & & & & \\
\hline Parat & $3.13(3.2)$ & $2.43(2.6)$ & $3.68(3.5)$ & $15.71 * * *$ & 0.04 \\
\hline $\begin{array}{l}\text { SA-45-total } \\
\text { (rango 0-180) }\end{array}$ & $24.6(23.9$ & $17.4(17.7)$ & $30.3(26.5)$ & $32.32 * * *$ & 0.07 \\
\hline
\end{tabular}

Nota. La prueba de $F$ se refiere a las diferencias entre varones y mujeres. ${ }^{*} p$ $<0.05 ; * * * p<0.001 ; \eta^{2}$ (eta ${ }^{2}$ parcial $)=$ tamaño del efecto $(\%$ de varianza explicada).

Tabla 4. Percentiles (puntos de corte) para las puntuaciones en las escalas SA-45.

\begin{tabular}{|c|c|c|c|c|c|c|c|c|c|c|c|c|c|c|c|c|c|c|c|c|}
\hline \multirow[t]{2}{*}{$\begin{array}{l}\text { Per- } \\
\text { centil }\end{array}$} & \multicolumn{2}{|c|}{ Depresión } & \multicolumn{2}{|c|}{ Hostilidad } & \multicolumn{2}{|c|}{$\begin{array}{c}\text { Sensibilidad } \\
\text { Interperso- } \\
\text { nal }\end{array}$} & \multicolumn{2}{|c|}{$\begin{array}{l}\text { Somatiza- } \\
\text { ción }\end{array}$} & \multicolumn{2}{|c|}{ Ansiedad } & \multicolumn{2}{|c|}{ Psicoticismo } & \multicolumn{2}{|c|}{$\begin{array}{l}\text { Obsesión } \\
\text { Compul- } \\
\text { sión }\end{array}$} & \multicolumn{2}{|c|}{$\begin{array}{l}\text { Ansiedad } \\
\text { Fóbica }\end{array}$} & \multicolumn{2}{|c|}{$\begin{array}{l}\text { Ideación } \\
\text { Paranoide }\end{array}$} & \multicolumn{2}{|c|}{$\begin{array}{l}\text { SA-45 } \\
\text { Total }\end{array}$} \\
\hline & $\pi$ & $q$ & ¿’ & q & $\pi$ & $q$ & $\delta^{1}$ & $q$ & $\pi$ & q & $\pi$ & q & $\pi$ & $q$ & $\delta$ & $q$ & $\pi$ & $q$ & त) & ㅇ \\
\hline 5 & 0 & 0 & 0 & 0 & 0 & 0 & 0 & 0 & 0 & 0 & 0 & 0 & 0 & 0 & 0 & 0 & 0 & 0 & 0 & 1 \\
\hline 10 & 0 & 0 & 0 & 0 & 0 & 0 & 0 & 0 & 0 & 0 & 0 & 0 & 0 & 0 & 0 & 0 & 0 & 0 & 1.6 & 3 \\
\hline 15 & 0 & 0 & 0 & 0 & 0 & 0 & 0 & 0 & 0 & 0 & 0 & 0 & 0 & 0 & 0 & 0 & 0 & 0 & 3 & 5 \\
\hline 20 & 0 & 0 & 0 & 0 & 0 & 0 & 0 & 0 & 0 & 0 & 0 & 0 & 0 & 1 & 0 & 0 & 0 & 0 & 4 & 7 \\
\hline 25 & 0 & 0 & 0 & 0 & 0 & 0 & 0 & 1 & 0 & 1 & 0 & 0 & 0.5 & 1 & 0 & 0 & 0 & 1 & 5 & 10 \\
\hline 30 & 0 & 1 & 0 & 0 & 0 & 1 & 0 & 1 & 0 & 1 & 0 & 0 & 1 & 2 & 0 & 0 & 0 & 1 & 6 & 11.2 \\
\hline 35 & 0 & 2 & 0 & 0 & 0 & 1 & 0 & 1 & 0 & 2 & 0 & 0 & 1 & 2 & 0 & 0 & 1 & 2 & 7 & 14 \\
\hline 40 & 1 & 2 & 0 & 0 & 0 & 1 & 1 & 2 & 0 & 2 & 0 & 0 & 1 & 3 & 0 & 0 & 1 & 2 & 8 & 18 \\
\hline 45 & 1 & 2.3 & 0 & 1 & 1 & 2 & 1 & 2 & 1 & 3 & 0 & 0 & 2 & 3 & 0 & 0 & 1 & 2 & 9.7 & 22 \\
\hline 50 & 1 & 3 & 0 & 1 & 1 & 2 & 1 & 3 & 1 & 3 & 0 & 0 & 2 & 4 & 0 & 0 & 2 & 3 & 11 & 26 \\
\hline 55 & 2 & 4 & 1 & 1 & 1 & 3 & 1 & 3 & 1 & 4 & 0 & 1 & 3 & 5 & 0 & 1 & 2 & 3 & 12 & 28 \\
\hline 60 & 2 & 4 & 1 & 2 & 2 & 3 & 2 & 4 & 2 & 4 & 0 & 1 & 3 & 5 & 0 & 1 & 2 & 4 & 15 & 30 \\
\hline 65 & 2.9 & 5 & 1 & 2 & 2 & 4 & 2 & 4 & 2 & 5 & 1 & 1 & 3 & 6 & 0 & 2 & 3 & 4 & 17 & 33.1 \\
\hline 70 & 3 & 5.8 & 2 & 3 & 2 & 4 & 2 & 4.8 & 2.2 & 6 & 1 & 2 & 4 & 7 & 1 & 2 & 3 & 5 & 20 & 37.8 \\
\hline 75 & 3 & 6 & 2 & 3 & 3 & 5.5 & 3 & 5 & 3 & 7 & 2 & 2 & 4.5 & 7 & 1 & 3 & 4 & 6 & 25 & 41.5 \\
\hline 80 & 4 & 7 & 3 & 5 & 3 & 7 & 3.8 & 6 & 4 & 8 & 2 & 2.2 & 5 & 8 & 1 & 4 & 4 & 7 & 29.6 & 49 \\
\hline 85 & 5 & 9 & 4 & 5 & 5 & 8 & 4 & 7 & 5 & 9 & 3 & 3 & 6 & 10 & 2 & 5 & 5 & 8 & 35 & 56.9 \\
\hline 90 & 6 & 11 & 6 & 8 & 5.4 & 9.6 & 5.4 & 9 & 6 & 1.6 & 4 & 4.6 & 8 & 11 & 3 & 7 & 6 & 9 & 46.2 & 71 \\
\hline 95 & 8 & 15.3 & 8.7 & 12 & 8 & 12 & 8 & 11.3 & 8 & 13 & 5 & 6 & 9 & 13.3 & 5 & 9.3 & 8 & 11 & 55.4 & 87 \\
\hline
\end{tabular}

Nota. Varones $\left(\delta^{\Uparrow}\right) n=216$; mujeres (Q) $\left.n=202\right)$; muestra total $(\hat{\delta}+\phi) N=418$.

Posteriormente, mediante la aplicación de análisis de varianza univariados, obtuvimos los efectos separados de la variable sexo sobre cada una de las nueve variables dependientes (véase la Tabla 3). Como puede observarse, los efectos fueron significativos para todas las escalas del SA-45 excepto para Psicoticismo. Se constatan, así mismo, que en todas estas escalas las puntuaciones eran significativamente superiores en el grupo de mujeres que en el de varones (incluida la escala Hostilidad), así como también en la puntuación total del cuestionario. Aunque el grueso de la literatura tiende a asociar puntuaciones más elevadas en la mujer que en el varón, tanto en el SA-45 (e.g., Davison et al., 1997; Sandín et al., 2008) como en el SCL-90-R (e.g., Cao, Fu, Wu y Chen, 2009; Derogatis, 1994; Kirkcaldy, Furnham y Siefen, 2010), en general estas diferencias suelen reducirse a algunas escalas, especialmente las relacionadas con ansiedad, depresión, somatización y sensibilidad interpersonal.

Finalmente, con fines descriptivos calculamos los percentiles (puntos de corte) para las nueve escalas del SA-45 y para la puntuación total en el cuestionario, separando los valores correspondientes a los grupos de varones y mujeres (véase la Tabla 5). 


\section{Discusión}

Mediante el presente estudio examinamos la estructura factorial y otras propiedades psicométricas del cuestionario SA45 en una muestra de población mexicana. Este cuestionario no sólo es mucho más breve que el SCL-90-R (presenta únicamente la mitad de los ítems de éste), sino que fue desarrollado con objeto de solventar algunos de los problemas que se han venido atribuyendo a este cuestionario, tales como la inconsistencia de la estructura factorial, el excesivo solapamiento entre las dimensiones que reduce su validez interna y discriminante, así como también la resultante elevada correlación entre las escalas. Un cuestionario más breve, consistente y equilibrado permite, sin duda, una utilización más rutinaria y efectiva en la práctica clínica y en la investigación psicopatológica. Dada la importancia que tiene la validez transcultural de los cuestionarios, pretendíamos obtener información sobre la estructura factorial, la fiabilidad y los datos normativos a partir de una muestra de estudiantes universitarios mexicanos aplicando el SA-45 validado previamente por Sandín et al. (2008).

En lo que concierne a la estructura factorial, nuestros datos confirman en general la estructura de nueve factores validada recientemente por Sandín et al. (2008). De los tres modelos examinados mediante técnicas de análisis factorial confirmatorio, el modelo de nueve factores correlacionados mostró índices de bondad de ajuste superiores a los restantes modelos (i.e., modelo de un factor general y modelo jerárquico), siendo menos relevantes (peor ajuste) otras alternativas que se han venido sugiriendo en la literatura sobre la estructura del SCL-90-R, tales como la predominancia de un solo factor general (e.g., Carlozzi y Long 2008; Olsen, Mortensen y Bech, 2004), la hipótesis sobre una estructura jerárquica basada en nueve factores primarios y dos factores de orden superior (distress general o neurosis y pensamiento paranoide o psicosis) (e.g., Arrindell, et al., 2006; Bados et al., 2005).

En general la estructura factorial es bastante coherente con la diferenciación de las nueve dimensiones, y es similar a la sugerida por Sandín et al. (2008). Algunos de los coeficientes de las estimaciones paramétricas parecen presentar valores menos relevantes (inferiores a .40), correspondiendo básicamente al factor de psicoticismo, lo cual es consistente

\section{Referencias}

Arrindell, W., Barelds, D., Janssen, I., Buwalda, F., y van der Ende, J. (2006). Invariance of SCL-90-R dimensions of symptom distress in patients with peri partum pelvic pain (PPPP) syndrome. British Journal of Clinical Psychology, 45, 377-391.

Bados, A., Balaguer, G., y Coronas, M. (2005). ¿Qué mide realmente el SCL-90-R? Estructura factorial en una muestra mixta de universitarios y pacientes. Psicología Conductual, 13, 181-196.

Barker-Collo, S. (2003). Culture and validity of the Symptom Checklist-90Revised and Profile of Mood States in a New Zealand student sample. Cultural Diversity and Ethnic Minority Psychology, 9, 185-196.

Belloch, A., Reina, N., García-Soriano, G., y Clark, D.A. (2009). Inventario Clark-Beck de Obsesión-Compulsión (C-BOCI): Validación para su uso con los datos presentados por estos autores. Aunque en principio nos llamó la atención el bajo valor del ítem 38 (ataque de temor o pánico) (Tabla 2), en realidad no debe sorprendernos ya que este ítem, aunque ha sido asignado al factor 5 (ansiedad), también satura en el factor 8 (ansiedad fóbica), factor este último más relacionado conceptualmente con la agorafobia y el pánico que con la ansiedad generalizada.

En lo que concierne a la fiabilidad (consistencia interna) del cuestionario, observamos que, con pequeñas excepciones, ésta es entre buena y excelente. Todas las escalas presentan valores satisfactorios de coeficientes alfa, salvo la escala de psicoticismo cuyo valor no alcanza el mínimo de .70. La baja frabilidad de esta escala también ha sido documentada en los estudios previos de Davison et al., (1997) y Sandín et al. (2008). Por tanto, el uso de esta escala como indicador del psicoticismo debe ser llevado a cabo con las debidas precauciones. Las correlaciones corregidas ítem-escala también apoyan, en general, la consistencia interna de las distintas dimensiones del cuestionario, siendo más bajas las correlaciones vinculadas a los items de la escala de psicoticismo.

Las puntuaciones medias en las distintas variables del cuestionario indican que, salvo en psicoticismo, los valores son siempre superiores en las mujeres que en los varones. Aunque en general las mujeres suelen puntuar más alto en muchas de las escalas del cuestionario (Davison et al., 1997; Sandín et al., 2008), nos ha llamado la atención que las mujeres puntúen significativamente más alto que los hombres en la escala de hostilidad, aunque tal vez podría explicarse porque esta escala sea más representativa de la agresividad verbal que de la física (la agresividad verbal suele ser superior en la mujer).

En suma, el presente estudio proporciona datos que corroboran y amplían la información referida por Sandín et al. (2008) sobre la versión española del SA-45. Aun tratándose de muestras culturalmente bien diferenciadas como la española y la mexicana, constatamos que las propiedades psicométricas del cuestionario son muy similares para ambos tipos de población, lo cual constituye una contribución de nuestro trabajo a la validez transcultural del SA-45. Futuros estudios deberán determinar la validez de este instrumento para diferenciar entre distintos síndromes o trastornos psicopatológicos. en población española. Revista de Psicopatología y Psicología Clínica, 14, 95105.

Bentler, P. M. (1992). On the fit of models to covariances and methodology to the Bulletin. Psychological Bulletin, 112, 400-404.

Bentler, P. M. (2005). EQS 6.1. for Windows. Structural equations program manual. Encino, CA: Multivariate Software, Inc.

Bollen, K. A. (1989). Structural equations with latent variables. New York: Wiley. Brown, T. A. (2006). Confirmatory factor analysis for applied research. New York: Guilford Press.

Cao, J., Fu, W., Wu, P., y Chen, D. (2009). Establishment of Symptom Checklist 90 Revised norm of Jiangsu Province. Chinese Journal of Clinical Psychology, 17, 681-683. 
Carlozzi, N., y Long, P. (2008). Reliability and validity of the SCL-90-R PTSD subscale. Journal of Interpersonal Violence, 23, 1162-1176.

Cattell, R. b. (1966). The scree test for the number of factors. Multivariate Behavioral Research, 3, 245-276.

Davison, M. K., Berhadsky, B., Bieber, J., Silversmith, D., Maruish, M. E., y Kane, R. L. (1997). Development of brief, multidimensional, self report instrument for treatment outcomes assessment in psychiatric settings: Preliminary finding. Assessment, 4, 259-276.

Delclaux, M. J. (2011). La niebla quimica: Efectos producidos por la quimioterapia en pacientes con cáncer (Ttrabajo de investigación Diploma de Estudios Avanzados). Madrid: UNED.

Derogatis, L. R. (1990). SCL-90-R: A bibliography of research reports 1975-1990. Baltimore, MD: Clinical Psychometric Research.

Derogatis, L. R. (1994). Symptom Checklist-90-R: Administration, scoring, and procedures manual. Minneapolis, Minn: National Computer Systems Inc.

Derogatis, L.R., Lipman, R.S., y Covi, L. (1973). SCL-90. An outpatient psychiatric rating scale - preliminary report. Psychopharmacological Bulletin, 9, $13-25$.

Derogatis, L.R, y Savitz, K.L. (2000). The SCL-90-R and brief symptom inventory (BSI) in primary care. En M. E. Maruish (Ed.), Handbook of Psychological Assessment in Primary Care Settings (pp. 297-334).; Mahwah, NJ: Lawrence Erlbaum Associates.

Fonseca, E., Paino, M., Lemos, S., y Muñiz, J. (2011). Prevalencia de la sintomatología emocional y comportamental en adolescentes españoles a través del Strengths and Dificulties Questionnaire (SDQ). Revista de Psicopatología y Psicología Clínica, 16, 15-25.

Gempp, F., R., y Avendaño, B., C. (2008). Datos Normativos y Propiedades Psicométricas del SCL-90-R en estudiantes universitarios chilenos. Terapia psicológica, 26, 39-58.

Gorsuch, R. (1983). Factor analysis. Hilsdale, NJ: Lawrence Erlbaum Associates.

Grill, S. S., Posada, M. C., y Castañeiras, C. (2009). Diferencias individuales, crecimiento postraumático y resiliencia ante situaciones traumáticas: El caso de los excombatientes de Malvinas. Anuario de Proyectos e Informes de Investigación de Becarios de Investigación, 6, 264-267.

Hard, J., Gerbershagen, H. U., y Franke, P. (2000). The symptom check-list, SCL-90-R: Its use and characteristics in chronic pain patients. European Journal of Pain, 4, 137-148.

Hu, L., y Bentler, P. M. (1999). Cutoff criteria for fit indexes in covariance structure analysis: Conventional criteria versus new alternatives. Structural Equation Modeling, 6, 1-15.

Jin, S., Yan, L., Li, B., Wen, T., Zhao, J., Zeng, Y., et al. (2010). Quality of life and psychologic distress of recipients after adult living-donor liver transplantation (LDLT)-A study from mainland China. Transplantation Proceedings, 42, 2611-6.

Kirkcaldy, B., Furnham, A., y Siefen, R. (2010). Social, family and psychological predictors of obsessive-compulsive behaviour among children and adolescents. School Psychology International, 31, 42-59.

López, A., Fernández, E., y Becoña, E. (2009). Comparación de las puntuaciones del SCL-90-R entre personas con dependencia de la nicotina y personas con dependencia de la cocaína al inicio del tratamiento. Revista de Psicopatología y Psicología Clínica, 14, 17-23.

Maruish, M. E. (2000). Applications of the Symptom Assessment-45 Questionnaire (SA-45) in primary care settings. En M. E. Maruish (Ed.), Handbook of Psychological Assessment in Primary Care Settings (pp. 335-372). Mahwah, NJ: Lawrence Erlbaum Associates.

Maruish, M. E., Bershadsky, B., y Golstein, L. (1998). Realiabity and validy of the SA-45: Further evidence from a primary care setting. Assesment, 5, 407-419.

Merino, O. (2010). Sindrome de burnout y variables de salud. Un estudio en profesionales sanitarios (Trabajo de investigación fin de máster). Madrid: UNED.

Nunnally, J. A. (1978). Psychometric theory. New York: McGraw-Hill.

Olsen, L.R., Mortensen, E.L., y Bech, P. (2004). The SCL-90 and SCL-90-R versions validated by ítem response models in a Danish community simple. Acta Psychiatrica Scandinavica, 110, 225-229.

Pilatti, A., Godoy, J.C., y Brussino, S.A. (2010). Construcción y valoración psicométrica del Cuestionario de Expectativas hacia el Alcohol para Adolescentes de Argentina (CEA-A). Anales de Psicología, 26, 288-301.

Sandín, B., Chorot, P., Santed, M.A., y Valiente, R.M. (2002). Análisis factorial confirmatorio del Índice de Sensibilidad a la Ansiedad para Niños. Psicothema, 14, 333-339.

Sandín, B., Chorot, P., Valiente, R.M., y Chorpita, B.F. (2010). Development of a 30-ítem version of the Revised Child Anxiety and Depression Scale. Revista de Psicopatología y Psicología Clínica, 15, 165-178.

Sandín, B., Chorot, P., Valiente, R.M., y Lostao, L. (2009). Validación española del cuestionario de preocupación PSWQ: Estructura factorial y propiedades psicométricas. Revista de Psicopatología y Psicología Clínica, 14, 107-122.

Sandín, B., Chorot, P., Valiente, R.M., Lostao, L., \& Santed, M.A. (2002). Adverse side effects in women attending a second stage breast cancer screening. Journal of Psychosomatic Research, 52, 303-309.

Sandín, B., Valiente, R.M., y Chorot, P. (2009). RCADS: Evaluación de los síntomas de los trastornos de ansiedad y depresión en niños y adolescentes. Revista de Psicopatología y Psicología Clínica, 14, 193-206.

Sandín, B., Valiente, R.M., Chorot, P., Sanded, M. A., y Lostao, L. (2008). SA-45: forma abreviada del SCL-90. Psicothema, 20, 290-296.

Schmitz, N., Hartkamp, N., Kiuse, J., Franke, G. H., Reister, G., y Tress, W. (2000). The Symptom Check-List-90-R (SCL-90-R): A German validation study. Quality of Life Research, 9, 185-193.

Strauman, T.J., y Wetzler, S. (1992). The factor structure of the SCL-90 and MCMI scale scores: within-measure and interbattery analyses. Multivariate Behavioral Research, 27, 1-20.

Vassend, O., y Skrondal, A. (1999). The problem of structural indeterminacy in multidimensional symptom report instruments: The case of SCL-90R. Behaviour Research and Therapy, 37, 685-701.

Wider, A. (1948). The Cornell Medical Index. New York: Psychological Corporation.

Zack, M., Toneatto, T., y Streiner, D.L. (1998). The SCL-90 factor structure in comorbid substance abusers. Journal of Substance Abuse, 10, 85-101.

(Articulo recibido: 29-03-2011, revisión: 31-08-2012, aceptado: 19-09-2012) 